\title{
Mortalidade por câncer de mama em mulheres indígenas brasileiras
}

ARTIGO ORIGINAL

\section{Descritores}

Neoplasias da mama

Epidemiologia

Mortalidade

População Indígena

Brasil

\section{Breast cancer mortality among Brazilian indigenous women}

Ruffo Freitas-Junior ${ }^{1,3}$, Leonardo Ribeiro Soares ${ }^{2}$, Carolina Maciel Reis Gonzaga ${ }^{3}$, Ana Luiza Lima Sousa ${ }^{4}$, Marilana Geimba de Lima ${ }^{5}$, Ludmilla Watanabe Branquinho ${ }^{6}$, Marta Rovery Souza ${ }^{7}$

\section{RESUMO}

Objetivo: Avaliar a mortalidade por câncer de mama em mulheres indígenas do Brasil, nos anos de 2000 e de 2010. Métodos: Trata-se de um estudo retrospectivo, realizado por meio de coleta de número de óbitos por câncer de mama em mulheres indígenas brasileiras, nos anos de 2000 e de 2010. Os dados foram obtidos por meio do Instituto Brasileiro de Geografia e Estatística e do Sistema de Informaçóes sobre Mortalidade. Foram calculadas as taxas de mortalidade bruta e a razão de risco $(\mathrm{RR})$ entre a mortalidade observada entre mulheres de cor branca e indígenas, considerando um intervalo de confiança de 95\% (IC95\%). Para o teste do $\chi^{2}$ com correção de Yates, valores de $\mathrm{p}<0,05$ foram considerados significativos. Resultados: No Brasil, a taxa bruta de mortalidade para mulheres indígenas foi de 4,72/100.000 em 2000, e de 2,23/100.000 em 2010. Na distribuição por macrorregiôes, observou-se que de 10 óbitos registrados em 2000, oito ocorreram na região Sudeste. Já em 2010, entre os cinco óbitos registrados, três ocorreram na regiāo Norte. As mulheres indígenas brasileiras apresentaram menor risco de mortalidade em relação às mulheres de cor branca, tanto em 2000 (RR: 0,25; IC95\% 0,138-0,47; p<0,001) quanto em 2010 (RR: 0,094; IC95\% 0,03-0,22; p<0,001). Conclusáo: A taxa de mortalidade por câncer de mama em mulheres indígenas no Brasil foi significantemente inferior ao observado em mulheres brancas, possivelmente em decorrência de variaçôes étnicas, geográficas e socioculturais. Esses dados podem contribuir para o desenvolvimento de estratégias direcionadas ao controle da neoplasia mamária na população indígena brasileira.

\section{ABSTRACT}

Objective: To evaluate mortality from breast cancer in Indigenous women in Brazil, in 2000 and 2010. Methods: This was a retrospective study, by means of collecting the number of breast cancer deaths among Brazilian women in the years 2000 and 2010. Data were obtained from the Brazilian Institute of Geography and Statistics and Mortality Information System. The crude mortality rates were calculated and the risk ratio (RR) of mortality observed among white women and indigenous women were calculated. It was used a confidence interval of $95 \%$ (C195\%). For the $\chi 2$ test with Yates correction, $p<0.05$ were considered significant. Results: The crude mortality rate for indigenous women was 4.72/100,000 in 2000 and 2.23/100,000 in 2010. In the distribution by geographical regions, it was observed that out

Trabalho realizado na Rede Goiana de Pesquisa em Mastologia - Goiânia (GO), Brasil .

${ }^{1}$ Hospital Araújo Jorge da Associação de Combate ao Câncer em Goiás (ACCG) - Goiânia (GO), Brasil.

Departamento de Ginecologia e Obstetrícia do Hospital das Clínicas da Universidade Federal de Goiás (UFG) - Goiânia (GO), Brasil

3Programa de Mastologia da UFG - Goiânia (GO), Brasil.

${ }^{4}$ Faculdade de Enfermagem da UFG - Goiânia (GO), Brasil.

${ }^{5}$ Setor de Mastologia da Secretaria Municipal de Saúde Pública de Campo Grande - Campo Grande (MS), Brasil.

${ }^{6}$ Faculdade de Medicina e membro da Liga da Mama da UFG - Goiânia (GO), Brasil.

${ }^{7}$ Instituto de Patologia Tropical e Saúde Pública da UFG - Goiânia (GO), Brasil.

Endereço para correspondência: Ruffo Freitas-Junior - Programa de Mastologia do Hospital das Clínicas da Universidade Federal de Goiás -

$1^{\text {a }}$ Avenida, s/n - Setor Universitário - CEP: 74605-050 - Goiânia (GO), Brasil - E-mail: ruffojr@terra.com.br

Conflito de interesses: nada a declarar.

Recebido em: 01/07/2015. Aceito em: 02/07/2015 
of 10 deaths recorded in 2000, eight occurred in the Southeast Region. In 2010, among the five reported deaths, three occurred in the North Region. Brazilian indigenous women had lower risk of breast cancer mortality compared to white women, both in 2000 (RR: 0.25; 95\%CI 0.138-0.47; $p<0.001$ ) and in 2010 (RR: 0.094, 95\%CI 0.03-0.22; $p<0.001$ ). Conclusion: The mortality rate from breast cancer among indigenous women in Brazil was significantly lower than in white women, possibly due to ethnic, geographic and socio-cultural variations. This data can contribute to the development of strategies aimed at controlling the breast cancer in Brazil's indigenous population.

\section{Introdução}

A melhoria dos serviços de informações sobre a mortalidade observada nos últimos anos ${ }^{1}$ foi acompanhada pelo aumento das estatísticas e do conhecimento acerca da mortalidade por câncer de mama no Brasil ${ }^{2,3}$. No entanto, considerando as diferentes origens étnicas e a miscigenação racial da população brasileira ${ }^{4}$, torna-se necessária a exploração dessas taxas de mortalidade em avaliaçóes específicas por subgrupo étnico.

Nesse contexto populacional, deve-se destacar a escassez de estudos nacionais envolvendo o câncer de mama em mulheres indígenas. Tradicionalmente, o perfil de mortalidade envolvendo a população indígena brasileira revelava prevalência de patologias infecciosas e parasitárias. Nos últimos anos, em decorrência da urbanização e da mudança dos hábitos de vida, tem-se observado aumento gradativo da mortalidade por doenças crônicas não transmissíveis, como diabetes e hipertensão arterial ${ }^{5-7}$.

Ao observar informaçóes da Fundação Nacional do Índio (FUNAI), nota-se que a população indígena tem apresentado variaçôes significativas nos últimos censos demográficos ${ }^{8}$. Na década de 1990, por exemplo, o contingente de brasileiros que se consideravam indígenas cresceu $150 \%$. Segundo dados do Instituto Brasileiro de Geografia e Estatística (IBGE), a população indígena brasileira em 2010 era composta por 817.963 indivíduos, dos quais 502.783 residiam na zona rural $^{8}$.

Embora a associação entre fatores de risco e a incidência de câncer de mama em mulheres indígenas tenha sido previamente observada ${ }^{9,10}$, não identificamos estudos acerca da mortalidade por neoplasia mamária nessa população. Dessa forma, o objetivo desse estudo foi avaliar a mortalidade por câncer de mama em mulheres indígenas, nos anos de 2000 e de 2010.

\section{Métodos}

Trata-se de um estudo retrospectivo, analítico, realizado por meio da coleta de número de óbitos por câncer de mama entre as mulheres brasileiras, nos anos de 2000 e de 2010. Os dados populacionais foram obtidos por meio do IBGE; e as informaçóes de óbitos por câncer de mama no Brasil foram coletadas através do Sistema de Informaçôes sobre Mortalidade (SIM), de acordo com a 10a revisão da Classificação Internacional de Doenças (CID C50).
Para os dados populacionais, optou-se pelos anos acima relatados pelo fato de serem anos censitários. Em decorrência do número reduzido de óbitos por câncer de mama na população indígena, optou-se por não utilizar as informaçôes intercensitárias, evitando dessa forma a possibilidade de viés metodológico relacionado à imprecisão da população total residente no Brasil.

Foram calculadas as taxas de mortalidade bruta por câncer de mama feminino de acordo com a cor, considerando as mulheres brancas e as mulheres indígenas separadamente. Também foram categorizadas por grupos etários, os quais foram categorizados em 20-49 anos e de 50 anos a mais. Os resultados foram avaliados pelas cinco macrorregiōes do país (Norte, Nordeste, Centro-Oeste, Sul e Sudeste). Para o cálculo da taxa bruta de mortalidade por câncer de mama por 100.000 mulheres, utilizou-se o quociente entre o total de mortes e a populaçáo sob risco. Utilizou-se a taxa bruta por não haver padronização mundial para a população indígena.

A análise estatística foi realizada com o auxílio do programa OpenEpi, versão 3.03a (Atlanta, USA). Foram elaboradas tabelas $2 \times 2$ para o cálculo da razão de risco entre a mortalidade observada em mulheres indígenas e mulheres de cor branca, considerando um intervalo de confiança de $95 \%$ (IC95\%). Para o teste do $\chi^{2}$ com correção de Yates, valores de $\mathrm{p}<0,05$ foram considerados significativos.

O estudo foi aprovado pelo Comitê de Ética em Pesquisa do Hospital das clínicas da Universidade Federal de Goiás (UFG), e faz parte de uma linha de pesquisa desenvolvida pela Rede Goiana de Pesquisa em Mastologia. Foram respeitadas todas as recomendaçôes de boas práticas clínicas segundo a resolução CNS 466/2012 e a Convenção de Helsinque.

\section{Resultados}

A população feminina residente e o total de óbitos por câncer de mama na população indígena, em 2000 e 2010, podem ser observados na Tabela 1. Em dez anos, apesar de um aumento de 5,8\% na população indígena, houve redução na taxa bruta de mortalidade por câncer de mama para essa raça. No período entre 2000 e 2010, foram registrados 82 óbitos de mulheres indígenas no Brasil, dos quais 34 (41,5\%) ocorreram na regiāo Sudeste e 19 (23,2\%) ocorreram na regiâo Nordeste. A evolução temporal do número de óbitos em mulheres indígenas, entre 2000 e 2010, pode ser observada na Tabela 2. 
Tabela 1. População residente, número total de óbitos e taxa bruta de mortalidade por câncer de mama em mulheres indígenas, no Brasil e macrorregiões, em 2000 e 2010

\begin{tabular}{lccc}
\hline \multirow{2}{*}{ Brasil e } & \multicolumn{3}{c}{2000} \\
\cline { 2 - 4 } & Populaçáo & $\begin{array}{c}\text { Óbitos por } \\
\text { câncer de mama }\end{array}$ & Taxa Bruta \\
Brasil & 211.839 & 10 & 4,72 \\
Norte & 46.683 & 1 & 2,14 \\
Nordeste & 51.690 & 1 & 1,93 \\
Sudeste & 60.088 & 8 & 13,31 \\
Sul & 26.229 & 0 & 0,00 \\
Centro-Oeste & 27.150 & 0 & 0,00 \\
& & 2010 & \\
Brasil & 224.219 & 5 & 2,23 \\
Norte & 66.726 & 3 & 4,50 \\
Nordeste & 63.040 & 0 & 0,00 \\
Sudeste & 39.753 & 2 & 5,03 \\
Sul & 22.274 & 0 & 0,00 \\
Centro-Oeste & 32.424 & 0 & 0,00 \\
\hline
\end{tabular}

Taxa Bruta: Taxa Bruta de mortalidade por 100.000 mulheres. Fontes: Sistema de Recuperaçáo Automática do Instituto Brasileiro de Geografia e Estatística (SIDRA/IBGE); e Sistema de Informação sobre Mortalidade (SIM/DATASUS).

Tabela 2. Número de óbitos em mulheres indígenas, segundo a macrorregião do Brasil, entre 2000 e 2010

\begin{tabular}{lcccccc}
\hline $\begin{array}{l}\text { Ano/ } \\
\text { Regiáo }\end{array}$ & Norte & Nordeste & Sudeste & Sul & $\begin{array}{c}\text { Centro- } \\
\text { Oeste }\end{array}$ & Total \\
2000 & 1 & 1 & 8 & 0 & 0 & 10 \\
2001 & 1 & 1 & 2 & 0 & 1 & 5 \\
2002 & 2 & 2 & 0 & 0 & 0 & 4 \\
2003 & 0 & 3 & 1 & 0 & 0 & 4 \\
2004 & 1 & 1 & 1 & 3 & 0 & 6 \\
2005 & 1 & 5 & 3 & 2 & 3 & 14 \\
2006 & 1 & 0 & 6 & 0 & 0 & 7 \\
2007 & 1 & 4 & 4 & 0 & 2 & 11 \\
2008 & 3 & 0 & 6 & 1 & 0 & 10 \\
\hline 2009 & 2 & 2 & 1 & 1 & 0 & 6 \\
2010 & 3 & 0 & 2 & 0 & 0 & 5 \\
Total & 16 & 19 & 34 & 7 & 6 & 82 \\
\hline
\end{tabular}

Fonte: Sistema de Informaçâo sobre Mortalidade (SIM/DATASUS).

As mulheres indígenas brasileiras apresentaram menor risco de mortalidade em relação às mulheres de cor branca, tanto em 2000 (RR: 0,25; IC95\% 0,138-0,47; p<0,001) quanto em 2010 (RR: 0,094; IC95\% 0,03-0,22; $\mathrm{p}<0,001)$. Na Tabela 3, pode ser observada a razão de risco de mulheres indígenas morrerem por câncer de mama em relação a mulheres brancas, na avaliaçãoo por grupo etário.

\section{Discussão}

A taxa de mortalidade por câncer de mama em mulheres indígenas brasileiras foi inferior ao observado em outras populaçóes ${ }^{2,3}$,
Tabela 3. Mortalidade por câncer de mama entre mulheres brancas e indígenas, de acordo com a idade, em 2000 e 2010

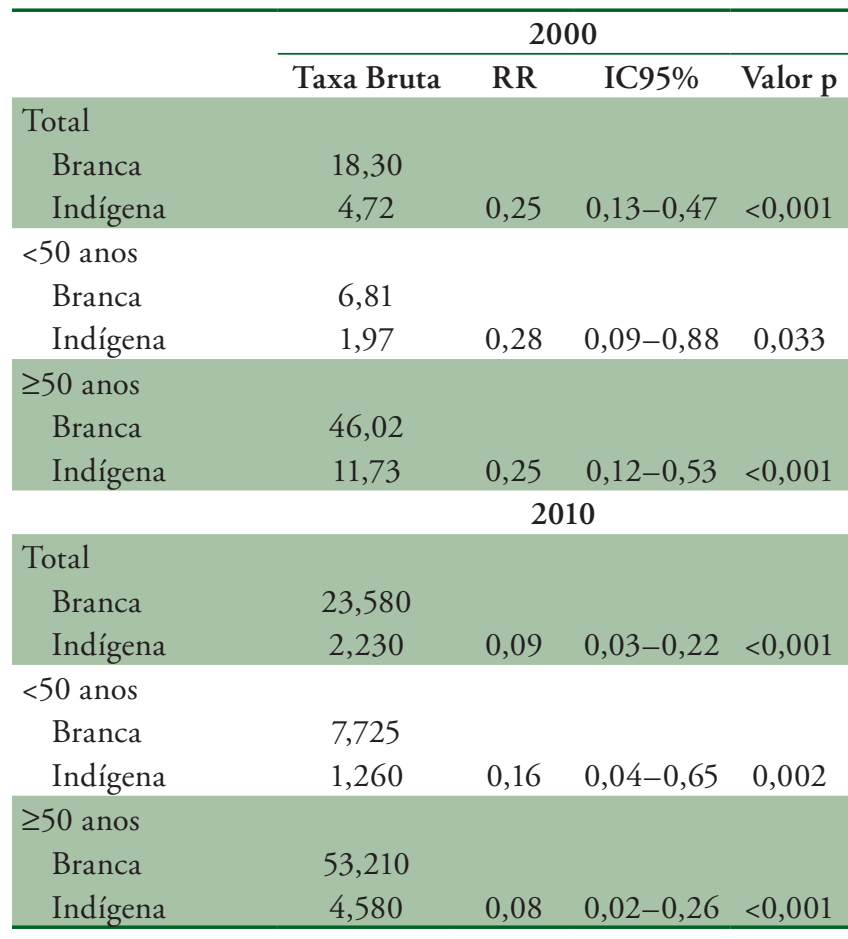

Taxa Bruta: Taxa Bruta de mortalidade por 100.000 mulheres; RR: razão de risco de mulheres indígenas morrerem por câncer de mama em relação à mulheres brancas; IC95\%: intervalo de confiança de 95\%.

Fonte: Sistema de Informação sobre Mortalidade (SIM/DATASUS).

o que pode ser decorrente do padrão de distribuição dos fatores de risco e da baixa incidência da neoplasia mamária nessa populaçãa $0^{9,10}$. Em estudos prévios, observou-se um perfil de baixa exposição hormonal e de redução no número total de ciclos ovulatórios, decorrentes de multiparidade e de aleitamento prolongado. Ainda, observaram-se gestaçôes em idade precoce e um padrão nutricional com predomínio de alimentos não industrializados, apesar da mudança nos hábitos de vida observada nos últimos anos ${ }^{9,10}$.

A densidade mamária é outro fator de risco que poderia contribuir para a baixa incidência da doença entre as mulheres indígenas. Nesse contexto, sabe-se que a presença de mamas densas pode reduzir a sensibilidade da mamografia, bem como se associar a determinados polimorfismos genéticos ${ }^{11,12}$. No entanto, não houve associação significativa entre a elevada prevalência de mamas não densas (82\%) em indígenas do Amapá com a expressão dos polimorfismos MspI do receptor de estrogênio e receptor de progesterona PROGINS ${ }^{13}$; o que torna necessário a realização de novos estudos envolvendo o perfil clínico e genético da população indígena brasileira.

Entre os demais fatores que poderiam justificar essa reduzida mortalidade em mulheres indígenas, destaca-se a subnotificação dos casos de câncer de mama. No entanto, estudos exploratórios realizados em populaçóes etnicamente determinadas observaram 
a ocorrência de neoplasias em outros sítios anatômicos, reduzindo a possibilidade de subnotificação do câncer de mama ${ }^{9,10}$.

Atualmente, postula-se que alteraçôes em genes que codificam proteínas envolvidas na biossíntese, na ação e no metabolismo dos esteroides sexuais poderiam se associar ao aumento da incidência e a diferentes padrōes de evolução clínica da doença ${ }^{14}$. Em estudos nacionais, foram observadas diferenças geográficas na distribuição dos subtipos moleculares do câncer de mama no Brasil ${ }^{15,16}$, sugerindo que fatores étnicos possam interferir em características específicas de biologia tumoral. Porém, a caracterização dessa origem gênica e a sua relação com a mortalidade por câncer de mama na população indígena brasileira ainda não foi estabelecida. Na maioria das etnias brasileiras existe uma organização social complexa e um modelo de cruzamento bem definido, criando um padrão genético que poderia se associar a fatores de proteção ao câncer de mama ${ }^{17}$.

No Brasil, na avaliação global da população indígena, deve ser considerada a heterogeneidade e o padrão de miscigenação das diversas etnias, bem como as suas características socioculturais $^{9,10,18}$. Os diferentes ancestrais genéticos podem determinar diferenças geográficas tanto no perfil genômico quanto na sua expressão fenotípica ${ }^{13,19,20}$. Essas variações podem influenciar as taxas de incidência do câncer de mama, e indiretamente, as taxas de mortalidade pela doença. Tais particularidades não foram analisadas no presente estudo, em decorrência da não disponibilidade no banco de dados utilizado.

Ao considerar-se a população de cor branca, observou-se nos últimos anos aumento da sobrevida em mulheres portadoras de câncer de mama, provavelmente em decorrência do diagnóstico precoce e de melhorias no tratamento da doença $a^{21,22}$. Entre as indígenas, um estudo prévio observou que o exame clínico das mamas e o rastreamento mamográfico são realizados de forma inconsistente ${ }^{10}$, o que tende a reduzir os benefícios do diagnóstico precoce nas taxas de mortalidade. Portanto, apesar da ausência de informaçóes acerca da sobrevida de indígenas brasileiras portadoras de câncer de mama, acredita-se que a consolidação do Sistema Único de Saúde (SUS) ${ }^{23,24}$ e o avanço do tratamento oncológico ${ }^{25}$ sejam fatores fundamentais para a manutençáo de baixas taxas de mortalidade nessa população.

Entre as variaçôes geográficas observadas no presente estudo, observou-se predomínio de óbitos na região Sudeste, provavelmente em decorrência da concentração populacional dessa região do país. Ainda, deve-se considerar o fator migratório, que compreende as pacientes que mudaram o Estado de residência em busca de melhor assistência à saúde ${ }^{26}$. No entanto, destaca-se o número reduzido de óbitos nas regiōes Sul e Centro-Oeste, as quais apresentam comunidades indígenas representativas ${ }^{9,10}$. Esses dados podem ser decorrentes da subjetividade acerca da definiçấo racial ${ }^{4}$ ou de variaçóes populacionais que ocorreram entre 2000 e 2010 no Brasil. Assim, acredita-se que uma das possibilidades da mudança na distribuição geográfica dos óbitos por câncer de mama em mulheres indígenas possa ser explicada pelas colocaçóes acima mencionadas.

Até onde sabemos, este foi o primeiro trabalho que analisou a mortalidade por câncer de mama em populaçáo indígena no Brasil, bem como sua comparação com a população de mulheres brancas. Dessa forma, o ineditismo reflete a importância das suas informaçōes. Já entre as limitaçóes, devem-se ressaltar as dificuldades envolvendo a qualidade das informaçôes coletadas em declaraçôes de óbito ${ }^{27}$; as quais não comprometem a credibilidade e a relevância do estudo atual. Ao considerar-se os tumores de maior prevalência, como o câncer de mama, observou-se uma elevada qualidade na geraçấo dos dados acerca da mortalidade no Brasil $1^{1}$. Portanto, diante da baixa cobertura dos Registros de Câncer de Base Populacional no território nacional, os dados do SIM representam uma importante fonte para a formulação de estratégias e políticas de saúde pública, devendo sempre ser utilizados.

Os resultados observados no presente estudo contribuem para o entendimento do processo saúde-doença envolvendo o câncer de mama entre as mulheres indígenas brasileiras, o qual é necessário para o desenvolvimento de estratégias específicas para o controle da neoplasia mamária nessa população.

\section{Conclusão}

Em 2000 e 2010, a taxa bruta de mortalidade por câncer de mama em mulheres indígenas brasileiras se mostrou significantemente inferior à observada para mulheres brancas, possivelmente em decorrência de subnotificação, do padrão de distribuição dos fatores de risco e da baixa incidência da neoplasia mamária nessa população.

\section{Referências}

1. Oliveira PPV, Silva GA, Curado MP, Malta DC, Moura L. Confiabilidade da causa básica de óbito por câncer entre Sistema de Informaçốes sobre Mortalidade do Brasil e Registro de Câncer de Base Populacional de Goiânia, Goiás, Brasil. Cad Saúde Pública. 2014;30(2):296-304

2. Gonzaga CMR, Freitas-Junior R, Souza MR, Curado MP, Freitas NMA. Disparities in female breast cancer mortality rates between urban centers and rural areas of Brazil: Ecological time-series study. Breast. 2014;23(2):180-7.

3. Gonzaga CMR, Freitas-Junior R, Curado MP, Sousa ALL, SouzaNeto JA, Souza MR. Temporal trends in female breast cancer mortality in Brazil and correlations with social inequalities: ecological time-series study. BMC Public Health. 2015;15:96.

4. Parra FC, Amado RC, Lambertucci JR, Rocha J, Antunes CM, Pena SD. Color and genomic ancestry in Brazilians. Proc Natl Acad Sci U S A. 2003;100(1):177-82.

5. Souza LG, Santos RV, Coimbra CE Jr. Estrutura etária, natalidade e mortalidade do povo indígena Xavante de Mato Grosso, Amazônia, Brasil. Ciênc Saúde Colet. 2010;15(Suppl 1):1465-73. 
6. Ferreira ME, Matsuo T, Souza RK. Aspectos demográficos e mortalidade de populaçōes indígenas do Estado do Mato Grosso do Sul, Brasil. Cad Saúde Pública. 2011;27(12):2327-39.

7. Schmidt MI, Duncan BB, Silva GA, Menezes AM, Monteiro CA, Barreto SM, et al. Chronic non-communicable diseases in Brazil: burden and current challenges. Lancet. 2011;377(9781):1949-61.

8. Fundação Nacional do Índio. Índios no Brasil: Quem são. [cited 2015 Jun 26]. Available from: http://www.funai.gov.br/index.php/ indios-no-brasil/quem-sao

9. Geimba de Lima M, Koifman S, Scapulatempo IL, Peixoto M, Naomi S, Curado do Amaral M. Fatores de risco para câncer de mama em mulheres indígenas Teréna de área rural, Estado do Mato Grosso do Sul, Brasil. Cad Saúde Pública. 2001;17(6):1537-44.

10. da Silva EP, Pelloso SM, Carvalho MD, Toledo MJ. Exploração de fatores de risco para câncer de mama em mulheres de etnia Kaingáng, Terra Indígena Faxinal, Paraná, Brasil, 2008. Cad Saúde Pública. 2009;25(7):1493-500.

11. Pettersson A, Graff RE, Ursin G, Santos Silva ID, McCormack V, Baglietto L, et al. Mammographic density phenotypes and risk of breast cancer: a meta-analysis. J Natl Cancer Inst. 2014;106(5). doi: 10.1093/jnci/dju078.

12. Mariapun S, Li J, Yip CH, Taib NA, Teo SH. Ethnic differences in mammographic densities: an asian cross-sectional study. PLoS One. 2015;10(2):e0117568. doi: 10.1371/journal.pone.0117568.

13. Secco JM, Nazário ACP, Elias S, Facina G, Araujo-Neto JT, Silva IDCG, et al. Densidade mamográfica e polimorfismos do gene do receptor de estrogênio $M s p I$ e do receptor de progesterona PROGINS nas índias do estado do Amapá. In: GBCS 2013: Anais do Goiania Breast Cancer Symposium; 2013 Mai 23-25; Goiânia, Brasil. Goiânia; 2013. p. 35.

14. Tokunaga E, Hisamatsu Y, Tanaka K, Yamashita N, Saeki H, Oki E, et al. Molecular mechanisms regulating the hormone sensitivity of breast cancer. Cancer Sci. 2014;105(11):1377-83.

15. Carvalho FM, Bacchi LM, Pincerato KM, Van de Rijn M, Bacchi CE. Geographic differences in the distribution of molecular subtypes of breast cancer in Brazil. BMC Womens Health. 2014;14:102.

16. de Macêdo Andrade AC, Ferreira Júnior CA, Dantas Guimarães B, Pessoa Barros AW, Sarmento de Almeida G, Weller M. Molecular breast cancer subtypes and therapies in a public hospital of Northeastern Brazil. BMC Womens Health. 2014;14:110.

17. Sztutman R. Do dois ao múltiplo na terra do um: a experiência antropológica de David Maybury-Lewis. Rev Antropol. 2002;45(2):443-76.

18. Kuhn PC, Horimoto AR, Sanches JM, Vieira Filho JP, Franco L, Fabbro AD, et al. Genome-wide analysis in Brazilian Xavante Indians reveals low degree of admixture. PLoS One. 2012;7(8):e42702. doi: 10.1371/journal.pone.0042702.

19. Alves-Silva J, da Silva Santos M, Guimarães PE, Ferreira AC, Bandelt HJ, Pena SD, et al. The ancestry of Brazilian mtDNA lineages. Am J Hum Genet. 2000;67(2):444-61.

20. Mendes-Junior CT, Simoes AL. Mitochondrial DNA variability among six South American Amerindian villages from the Pano linguistic group. Hum Biol. 2014;86(2):93-104.

21. Nunes RD. Estudo de sobrevida das mulheres com câncer de mama na cidade de Goiânia, entre 1995 e 2003 [Dissertação]. Goiânia: Universidade Federal de Goiás. Faculdade de Medicina; 2011.

22. Höfelmann DA, Anjos JC, Ayala AL. Sobrevida em dez anos e fatores prognósticos em mulheres com câncer de mama em Joinville, Santa Catarina, Brasil. Ciênc Saúde Colet. 2014;19(6):1813-24.

23. Santos NR. The evolution of the Brazilian National Health System, strategic courses of action and strategies to understand these actions. Ciênc Saúde Colet. 2007;12:429-35.

24. Atun R, de Andrade LO, Almeida G, Cotlear D, Dmytraczenko T, Frenz $\mathrm{P}$, et al. Health-system reform and universal health coverage in Latin America. Lancet. 2015;385(9974):1230-47.

25. Lee BL, Liedke PE, Barrios CH, Simon SD, Finkelstein DM, Goss PE. Breast cancer in Brazil: Present status and future goals. Lancet Oncol. 2012;13(3):e95-102.

26. Freitas-Junior R, Gonzaga CMR, Freitas NMA, Martins E, Dardes RCM. Disparities in female breast cancer mortality rates in Brazil between 1980 and 2009. Clinics. 2012;67(7):731-7.

27. Felix JD, Zandonade E, Amorim MHC, Castro DS. Avaliação da completude das variáveis epidemiológicas do Sistema de Informação sobre Mortalidade em mulheres com óbitos por câncer de mama na Região Sudeste - Brasil (1998 a 2007). Ciênc Saúde Colet. 2012;17(4):945-53. 